\title{
THE REPRESENTATION OF LATTICES BY MODULES
}

\author{
BY GEORGE HUTCHINSON \\ Communicated by Saunders Mac Lane, August 8, 1972
}

1. A quasivariety characterization of lattices representable by $\Lambda$-modules. If $\Lambda$ is a nontrivial ring with 1 , a lattice $L$ is "representable by $\Lambda$-modules" if it can be embedded in the lattice of submodules of some unitary left $\Lambda$-module $M$. This lattice of submodules is denoted $\Gamma(M ; \Lambda)$.

A (lattice) "Horn formula" is an open formula:

$$
\left(e_{1}=e_{2} \& e_{3}=e_{4} \& \ldots \& e_{n-3}=e_{n-2}\right) \Rightarrow e_{n-1}=e_{n},
$$

where $e_{1}, e_{2}, \ldots, e_{n}$ are lattice polynomials.

MAIN THEOREM. For every commutative ring $\Lambda$, there exists a set $J(\Lambda)$ of Horn formulas such that a lattice $L$ is representable by $\Lambda$-modules if and only if every formula of $J(\Lambda)$ is satisfied in L. Each member of $J(\Lambda)$ is constructible by a finite sequence of four basic operations.

That is, the class $\mathscr{L}(\Lambda)$ of lattices representable by $\Lambda$-modules is the "quasivariety" of lattices satisfying $\mathrm{J}(\Lambda)$, for commutative $\Lambda$.

OUTLINE OF PROOF. For $\Lambda$ commutative, let $\imath: L \rightarrow \Gamma(M ; \Lambda)$ be an embedding for some $M$. Without loss of generality, assume that $L$ has a smallest element $\omega$, and $\imath(\omega)=0$. Motivated by the "abelian" lattice $\Gamma_{f}\left(G^{N}\right)$ of $[2,4.2]$ with $G=M$, we consider "constraint systems" in variables $a_{k}$ (corresponding to coordinate positions in $M^{\mathbf{N}}$ ) and "auxiliary" variables $b_{k}$ (with existential quantifiers understood) for $k$ in $\boldsymbol{N}=\{1,2,3, \ldots\}$. Consider $\boldsymbol{r}=\left(d_{1}, d_{2}, d_{3}, d_{4}\right)$ below.

$\left(d_{1}\right) \quad a_{1} \in x_{1}, \quad a_{2} \in x_{2}, \quad a_{k} \in \omega$ for $k \geqq 3\left(x_{1}, x_{2} \in L\right)$.

$\left(d_{2}\right) \quad b_{1} \in x_{3}, \quad b_{2} \in x_{1}, \quad b_{k} \in \omega$ for $k \geqq 3\left(x_{3} \in L\right)$.

$\left(d_{3}\right) \quad a_{1}-a_{2}-b_{1}=0$.

$\left(d_{4}\right) \quad a_{1}-\lambda_{0} b_{2}=0 \quad\left(\lambda_{0} \in \Lambda\right)$.

A "solution" $f: \boldsymbol{N} \rightarrow M$ of $\boldsymbol{r}$ satisfies

$\left(e_{1}\right) \quad f(1) \in l\left(x_{1}\right), \quad f(2) \in l\left(x_{2}\right), \quad f(k) \in l(\omega)=0 \quad$ for $k \geqq 3\left(d_{1}\right)$.

$\left(e_{2}\right) \quad f(1)-f(2) \in l\left(x_{3}\right) \quad\left(d_{3}, b_{1} \in x_{3}\right)$.

$\left(e_{3}\right) \quad$ There exists $v \in l\left(x_{1}\right)$ such that $\lambda_{0} v=f(1)\left(d_{4}, b_{2} \in x_{1}\right)$. 
Formally, let $\boldsymbol{N}_{1}=\left\{a_{k}: k \in N\right\}$, let $\boldsymbol{N}_{2}=\boldsymbol{N}_{1} \cup\left\{b_{k}: k \in \boldsymbol{N}\right\}$, and let $M_{1}^{\infty}$ and $M_{2}^{\infty}$ be the $\Lambda$-modules of all functions $N_{1} \rightarrow M$ and $N_{2} \rightarrow M$, respectively. Let a " $\Lambda$-equation" be a function $g: N_{2} \rightarrow \Lambda$ such that $g\left(a_{k}\right)=g\left(b_{k}\right)=0$ except for finitely many $k$ in $N ; g$ determines the "linear solution set" $g^{*}$ in $\Gamma\left(M_{2}^{\infty} ; \Lambda\right)$ :

$$
g^{*}=\left\{m \in M_{2}^{\infty}: \sum_{k=1}^{\infty}\left(g\left(a_{k}\right) m\left(a_{k}\right)+g\left(b_{k}\right) m\left(b_{k}\right)\right)=0\right\}
$$

A "constraint function" is a function $\alpha: N_{2} \rightarrow L$ such that $\alpha\left(a_{k}\right)=\alpha\left(b_{k}\right)=\omega$ except for finitely many $k$; it determines a "box" $l_{*}(\alpha)$ in $\Gamma\left(M_{2}^{\infty} ; \Lambda\right)$ :

$$
\imath_{*}(\alpha)=\left\{m \in M_{2}^{\infty}: m\left(c_{k}\right) \in l \alpha\left(c_{k}\right) \text { for } c_{k} \in N_{2}\right\} .
$$

If $\alpha$ is a constraint function and $G=\left\{g_{1}, g_{2}, \ldots, g_{n}\right\}$ is a finite (possibly empty) set of $\Lambda$-equations, the pair $(G, \alpha)$ is a "constraint system". An "extended solution" $m: N_{2} \rightarrow M$ of $(G, \alpha)$ is a member of

$$
\mu_{0}(G, \alpha)=l_{*}(\alpha) \cap g_{1}^{*} \cap g_{2}^{*} \cap \cdots \cap g_{n}^{*} \text { in } \Gamma\left(M_{2}^{\infty} ; \Lambda\right) .
$$

A "solution" $m^{\prime}: N_{1} \rightarrow M$ of $(G, \alpha)$ is a restriction $m^{\prime}=m \mid N_{1}$ of an extended solution $m$. Let $D(L ; \Lambda)$ denote the set of all constraint systems. Given $M$ and $l$, define $\mu: D(L ; \Lambda) \rightarrow \Gamma\left(M_{1}^{\infty} ; \Lambda\right)$ by the "solution set" $\mu(G, \alpha)=\left\{m \mid N_{1}: m \in \mu_{0}(G, \alpha)\right\}$. Since $\imath(\omega)=0, \mu(G, \alpha)$ has "finite support" as in $[2$, p. 181].

Now, $D(L ; \Lambda)$ can be defined for any lattice $L$, not just those in $\mathscr{L}(\Lambda)$. Meet and join operations, corresponding to solution set intersection and sum, can be defined abstractly in $D(L ; \Lambda)$. We can also define "equivalence" of constraint systems, obtaining a congruence $E(L ; \Lambda)$ on $D(L ; \Lambda)$. If an embedding $\imath: L \rightarrow \Gamma(M ; \Lambda)$ with $l(\omega)=0$ exists, the corresponding $\mu: D(L ; \Lambda) \rightarrow \Gamma\left(M_{1}^{\infty} ; \Lambda\right)$ preserves meet and join and takes equivalent constraint systems modulo $E(L ; \Lambda)$ into the same solution set. Seven "rules of equivalence" generate $E(L ; \Lambda)$; we reconsider $r=$ $\left(d_{1}, d_{2}, d_{3}, d_{4}\right)$ to suggest them:

Constraint decrease: The lattice constraint of $a_{1}$ can be changed to $x_{1} \wedge\left(x_{2} \vee x_{3}\right)$, since $d_{3}$ can be solved for $a_{1}, a_{1}=a_{2}+b_{1}$, and $a_{2}+b_{1}$ is in $x_{2} \vee x_{3}$. Linear combination augmentation: Any $\Lambda$-equation of the form $\lambda d_{3}+\lambda^{\prime} d_{4}$ can be added to $r$. Defined variable augmentation: We can "define" an unused auxiliary variable, say $b_{4}$, by adding a $\Lambda$-equation, say $\lambda a_{2}+\lambda^{\prime} b_{2}+b_{4}=0$, if we change the lattice constraint of $b_{4}$ to $x_{2} \vee x_{1}\left(-\lambda a_{2}-\lambda^{\prime} b_{2}\right.$ is in $\left.x_{2} \vee x_{1}\right)$. Union augmentation: Add the $\Lambda$-equation $a_{2}-b_{7}-b_{9}=0$, for example, expressing a variable $a_{2}$ as a sum of two unused auxiliary variables. Then change the lattice constraints of $b_{7}$ and $b_{9}$ to some $x_{4}$ and $x_{5}$ in $L$, respectively, such that $x_{2} \subset x_{4} \vee x_{5}\left(a_{2} \in x_{2}\right)$. Null variable augmentation: Terms $\lambda a_{5}$ and $\lambda^{\prime} a_{5}$ 
can be added to the $\Lambda$-equations $d_{3}$ and $d_{4}$, respectively, since $a_{5} \in \omega$ and $l(\omega)=0$. Inessential variables augmentation: We can add finitely many $\Lambda$-equations in the variables $b_{k}, k \geqq 3$, and make finitely many arbitrary changes in the lattice constraints of those variables. Renumbering: $b_{1}$ and $b_{2}$ can be replaced throughout $\boldsymbol{r}$ by any two other auxiliary variables.

The solution set of $\boldsymbol{r}$ is unchanged by any of the above modifications. The primary fact about $M(L ; \Lambda)=D(L ; \Lambda) / E(L ; \Lambda)$ is that it is abelian lattice under the induced meet and join. Intuitively, $M(L ; \Lambda)$ acts like the lattice of submodules with finite support of $M^{N}$, for some hypothetical $\Lambda$-module $M$.

Associated with any abelian lattice $X$ is a small abelian category $\boldsymbol{A}_{X}$ [2, Main Theorem]. We next construct for each object $A$ of $\boldsymbol{A}_{M(L ; \Lambda)}$ a ring homomorphism $\zeta_{A}$ preserving 1 from $\Lambda$ into the ring of endomorphisms of $A\left(\zeta_{A}(\lambda)\right.$ is a formal analogue of $\left.\lambda 1_{A}\right)$. Let $\mathbf{A b}$ and $\Lambda$-Mod be the usual categories of abelian groups and of $\Lambda$-modules, respectively. By $[\mathbf{1}$. Theorem 7.14], there exists an exact embedding functor $F: \mathbf{A}_{M(L ; \Lambda)} \rightarrow \mathbf{A b}$. Defining $\lambda x=\left(F\left(\zeta_{A}(\lambda)\right)\right)(x)$ makes $F(A)$ into a $\Lambda$-module, denoted $G(A)$ $\left(F \zeta_{A}(\lambda)=\lambda 1_{G(A)}\right)$. We can prove that $\zeta_{B}(\lambda) f=f \zeta_{A}(\lambda)$ for $f: A \rightarrow B$ in $A_{M(L ; \Lambda)}$, so $F f: G(A) \rightarrow G(B)$ is $\Lambda$-linear. But then $G(A)$ and $G f=F f$ define an exact embedding functor $G: A_{M(L ; \Lambda)} \rightarrow \Lambda$-Mod. Because of $G$, the lattice of subobjects of each object of $A_{M(L ; \Lambda)}$ is in $\mathscr{L}(\Lambda)$. But then every interval sublattice of $M(L ; \Lambda)$ is in $\mathscr{L}(\Lambda)$ by $[2,3.24]$, and $M(L ; \Lambda) \in \mathscr{L}(\Lambda)$ follows, using a direct limit of $\Lambda$-modules.

We now define a lattice homomorphism $\psi: L \rightarrow M(L ; \Lambda)$, similar to $\psi$ in $[2,4.3]$. For $x$ in $L, \psi(x)$ is the equivalence class in $M(L ; \Lambda)$ of $\left(\varnothing, \theta_{x}\right)$ in $D(L ; \Lambda)$ given by $\theta_{x}\left(a_{1}\right)=x, \theta_{x}\left(c_{k}\right)=\omega$ for $c_{k} \in N_{2}-\left\{a_{1}\right\}$. If $\psi$ is one-to-one, it embeds $L$ into $M(L ; \Lambda)$, and so $L$ is in $\mathscr{L}(\Lambda)$. Suppose $L$ is in $\mathscr{L}(\Lambda)$ with embedding $l: L \rightarrow \Gamma(M ; \Lambda), \imath(\omega)=0$. Since equivalent constraint systems have equal solution sets, $\mu: D(L ; \Lambda) \rightarrow \Gamma\left(M_{1}^{\infty} ; \Lambda\right)$ induces a function $\bar{\mu}: M(L ; \Lambda) \rightarrow \Gamma\left(M_{1}^{\infty} ; \Lambda\right)$. Clearly $\bar{\mu} \psi(x)=\mu\left(\varnothing, \theta_{x}\right)=$ $\overline{\psi l}(x)$, where $\psi: \Gamma(M ; \Lambda) \rightarrow \Gamma\left(M_{1}^{\infty} ; \Lambda\right)$ is given by

$$
\bar{\psi}\left(M^{\prime}\right)=\left\{m \in M_{1}^{\infty}: m\left(a_{1}\right) \in M^{\prime}, m\left(a_{k}\right)=0 \text { for } k>1\right\} .
$$

So, $\bar{\psi}_{l}=\bar{\mu} \psi$. Since $\bar{\psi}_{l}$ is one-to-one, so is $\psi$. Therefore, $L$ is in $\mathscr{L}(\Lambda)$ if and only if $\psi$ is one-to-one.

Four of the rules generating $E(L ; \Lambda)$ are called "direct reductions", namely constraint decrease, linear combination augmentation, defined variable augmentation and union augmentation. A key argument shows that $\psi$ is one-to-one iff, for each $x$ in $L$ and sequence $\boldsymbol{r}_{1}, \boldsymbol{r}_{2}, \ldots, \boldsymbol{r}_{n}$ in $D(L ; \Lambda)$ such that $\boldsymbol{r}_{1}=\left(\varnothing, \theta_{x}\right), \boldsymbol{r}_{n}=(G, \alpha)$ and $\boldsymbol{r}_{i+1}$ is obtained by a direct reduction of $r_{i}(1 \leqq i<n)$, we have $\alpha\left(a_{1}\right)=x$. Each of the infinitely 
many Horn formulas of $J(\Lambda)$ is generated by a finite sequence of four operations. These operations imitate the four rules of direct reduction, with lattice polynomials replacing elements of $L$. Using the above, we show that $\psi$ is one-to-one iff every formula of $J(\Lambda)$ is satisfied in $L$, and the main theorem follows.

COROLLARY. Every abelian lattice is representable by abelian groups.

2. Comparison of classes of representable lattices. Let $\Lambda$ and $\Lambda^{\prime}$ be rings with 1 , not necessarily commutative. Then $\mathscr{L}(\Lambda) \subset \mathscr{L}\left(\Lambda^{\prime}\right)$ if there exists a ring homomorphism $\Lambda^{\prime} \rightarrow \Lambda$ preserving 1 , or if there exists a $\left(\Lambda^{\prime}, \Lambda\right)$ bimodule $M$ which is faithfully flat as a right $\Lambda$-module. A simple change of rings argument proves the first result. For the other: the exact embedding functor $M \otimes_{\Lambda}$ from $\Lambda$-Mod into $\Lambda^{\prime}$-Mod induces an embedding from the lattice of subobjects of any $M_{0}$ in $\Lambda$-Mod into the lattice of subobjects of $M \otimes_{\Lambda} M_{0}$ in $\Lambda^{\prime}$-Mod. Then $\mathscr{L}(\Lambda)=\mathscr{L}\left(\Lambda^{\prime}\right)$ if $\Lambda$ is a regular ring and unitary subring of $\Lambda^{\prime}$, by known ring theory. Let $Q$ denote the field of rationals and $Z_{n}$ the ring of integers modulo $n, n \geqq 2$. So, $\mathscr{L}(\Lambda)=$ $\mathscr{L}(\boldsymbol{Q})$ if $\Lambda$ has a unitary subring isomorphic to $\boldsymbol{Q}$. Also, $\mathscr{L}(\Lambda)=\mathscr{L}\left(\boldsymbol{Z}_{n}\right)$ if $\Lambda$ has characteristic $n$ for $n$ a square-free number (prime, or a product of distinct primes). Let $P_{\Lambda}$ be the set of primes $p$ such that $1+1+\cdots+1$ ( $p$ times) is invertible in $\Lambda$. If $P$ is a set of primes, let $Q(P)$ be the unitary subring of $Q$ generated by $\left\{p^{-1}: p \in P\right\}$. If $\Lambda$ has characteristic zero, $a$ is the two-sided ideal of torsion elements of $\Lambda$ and $P_{\Lambda / \mathfrak{a}}=P_{\Lambda}$, then $\mathscr{L}(\Lambda)=$ $\mathscr{L}\left(Q\left(P_{\Lambda}\right)\right)$. So, $\mathscr{L}(\Lambda)=\mathscr{L}\left(Q\left(P_{\Lambda}\right)\right)$ if $\Lambda$ is torsion-free.

Some of the above results are the best possible. Under various hypotheses, $\mathscr{L}(\Lambda)-\mathscr{L}\left(\Lambda^{\prime}\right) \neq \varnothing$ is proved by constructing a Horn formula satisfied in all lattices in $\mathscr{L}\left(\Lambda^{\prime}\right)$ but not in all lattices in $\mathscr{L}(\Lambda)$. These formulas reflect properties of the (additive) multiples $k \cdot 1_{M}=1_{M}+1_{M}+$ $\cdots+1_{M}$ for $M$ an arbitrary $\Lambda$-module. For example, $k \cdot 1_{M}=0$ if the characteristic of $\Lambda$ divides $k$, and $k \cdot 1_{M}$ is an automorphism if $k \cdot 1$ is invertible in $\Lambda$. So, we can show that $\mathscr{L}(\Lambda)-\mathscr{L}\left(\Lambda^{\prime}\right) \neq \varnothing$ if the characteristic of $\Lambda$ does not divide the (nonzero) characteristic of $\Lambda^{\prime}$, and therefore $\mathscr{L}(\Lambda) \neq \mathscr{L}\left(\Lambda^{\prime}\right)$ if $\Lambda$ and $\Lambda^{\prime}$ have different characteristics. If $p$ is a prime invertible in $\Lambda^{\prime}$ but not in $\Lambda$, then $\mathscr{L}(\Lambda)-\mathscr{L}\left(\Lambda^{\prime}\right) \neq \varnothing$, and so $\mathscr{L}(\Lambda) \neq$ $\mathscr{L}\left(\Lambda^{\prime}\right)$ if $P_{\Lambda} \neq P_{\Lambda^{\prime}}$. If $n$ is not square-free, then there exists $\Lambda$ with characteristic $n$ such that $\mathscr{L}(\Lambda) \neq \mathscr{L}\left(\boldsymbol{Z}_{n}\right)$. Also, if $\Lambda$ has characteristic zero and torsion ideal a, then $\mathscr{L}(\Lambda) \neq \mathscr{L}\left(Q\left(P_{\Lambda}\right)\right)$ if $P_{\Lambda / \mathfrak{a}} \neq P_{\Lambda}$. If $P$ is a proper subset of the primes or is empty, then $\Lambda$ with characteristic zero exists such that $P_{\Lambda}=P$ but $\mathscr{L}(\Lambda) \neq \mathscr{L}(Q(P))$.

The detailed proofs of these results have been submitted for publication.

C. Herrmann and W. Poguntke have recently communicated to the author a theorem which implies that $\mathscr{L}(\Lambda)$ admits ultraproducts, for 
any ring $\Lambda$ with 1 . It then follows nonconstructively that $\mathscr{L}(\Lambda)$ is always a quasivariety, using the known result that a class of algebras admitting isomorphic images, subalgebras, products and ultraproducts is a quasivariety. Another of their results implies that $\mathscr{L}(\Lambda)$ is not finitely firstorder axiomatizable if $\Lambda$ is a unitary subring of $Q$.

\section{REFERENCES}

1. P. J. Freyd, Abelian categories: An introduction to the theory of functors, Harper's Series in Modern Math., Harper \& Row, New York, 1964. MR 29 \# 3517.

2. G. Hutchinson, Modular lattices and abelian categories. J. Algebra 19 (1971), 156-184. MR 43 \# 4880.

Division of Computer Research and Technology, NiH, Public Health Service, Department of Health, Education and Welfare, Bethesda, Maryland 20014 